Fukushima J. Med. Sci.,

Vol. 61, No. 1, 2015

[Original Article]

\title{
STENTING STRATEGY AND FOLLOW-UP RESULTS OF MULTI-CENTER REGISTRY IN FUKUSHIMA CITY FOR LEFT MAIN CORONARY ARTERY DISEASE : BARE METAL STENT VERSUS DRUG-ELUTING STENT
}

\author{
KAZUHIKO NAKAZATO ${ }^{1}$, HIROYUKI MIZUKAMI ${ }^{1,2}$, HIDEKI OHTAKE ${ }^{3}$, \\ NOBUO SAKAMOTO ${ }^{1}$, TAKAYOSHI YAMAKI ${ }^{1}$, OSAMU YAMAGUCHI ${ }^{4}$, HIROYUKI KUNII $^{1}$, \\ TAKAYUKI OHWADA ${ }^{5}$ and YASUCHIKA TAKEISHI ${ }^{1}$ \\ ${ }^{1}$ Department of Cardiology and Hematology, Fukushima Medical University, ${ }^{2} J$ usendo General Hospital, \\ ${ }^{3}$ Saiseikai Fukushima General Hospital, ${ }^{4}$ Ohara Medical Center, ${ }^{5}$ Fukushima Red Cross Hospital
}

(Received February 13, 2015, accepted May 22, 2015)

\begin{abstract}
An appropriate treatment strategy for left main trunk (LMT) lesions is still controversial in the drug-eluting stent (DES) era. Consecutive LMT stenting cases $(n=155)$ between January 2008 and January 2013 in 4 hospitals in Fukushima city were retrospectively analyzed. We excluded the patients suffering from cardiogenic shock before the stenting procedure. Among those cases, 60 patients had acute coronary syndrome, and remaining 95 had stable angina pectoris. Out of 155 cases, 45 patients were treated with bare metal stents (BMSs) and 110 patients were treated with DESs. All cases were succeeded in the initial procedure. Mean stent size of BMS was 3.85 $\pm 0.34 \mathrm{~mm}$ while that of DES was $3.46 \pm 0.17 \mathrm{~mm}(P<0.001)$. At the follow up coronary angiography (255-day on average), \% stenosis of BMS group was $26.6 \pm 15.0 \%$ and that of DES group was $20.4 \pm 12.6 \%(P=0.006)$. The mean observation period for clinical events was $738.8 \pm 480.3$ days. Major adverse cardiac events-free rates for each group were compared and no significant differences were evident between the 2 groups (11.1\% vs. $19.1 \%$, ns). The present study demonstrated that use of BMSs would be a viable option in the treatment of LMT lesions when it is possible to use a large-sized stent ( $>3.5 \mathrm{~mm})$.
\end{abstract}

Key words : Left main trunk, Coronary stents, PCI, Fukushima city

\section{INTRODUCTION}

A left main trunk (LMT) lesion is an indication for coronary artery bypass graft (CABG) surgery in patients with ischemic heart disease (IHD), while percutaneous coronary intervention $(\mathrm{PCI})$ was previously contraindicated in unprotected LMT lesions $^{1)}$. Compared to today's standards, treatment outcomes for PCI to LMT were obviously poor when plain old balloon angioplasty (POBA) was the only available intervention ${ }^{2)}$. However, with the emergence of new devices, such as directional coronary atherectomy (DCA) catheters and coronary stents, and with the establishment of antiplatelet therapy, forward-thinking physicians began performing PCI even on unprotected LMT lesions ${ }^{3,4)}$. The established use of coronary stents in particular led to reports of PCI's superiority in terms of time to reperfusion in acute myocardial infarction (AMI) patients with hemodynamic instability, resulting in the procedure being included in treatment guidelines ${ }^{5,6)}$. Even after undergoing emergent CABG, AMI patients with LMT occlusion who go into shock have a very low survival rate. This set of circumstances yielded empirical evidence on the use of LMT catheterization mainly in patients with acute coronary syndrome (ACS). In recent years, the advent of drug-eluting stents (DESs) that drastically reduce

Corresponding author: Kazuhiko Nakazato E-mail : nakazato@fmu.ac.jp

https://www.jstage.jst.go.jp/browse/fms http://www.fmu.ac.jp/home/lib/F-igaku/ 
the risk of restenosis leading to expectations of better treatment outcomes and a series of studies on the use of PCI to treat LMT lesions ${ }^{7-9)}$. However, it has been pointed out that first-generation DESs can also cause problems, such as late-onset thrombosis following withdrawal from antiplatelet therapy, so a consensus has yet to be reached on the suitability of DESs in the treatment of $\mathrm{LMT}^{10)}$, in which procedural problems can be life threatening.

Elective PCI for LMT is still relatively contraindicated in principle in Japanese treatment guidelines, and if it will be performed, consultation with "Heart team" has been recommended ${ }^{11)}$. Therefore, accurate assessment of treatment outcomes is needed in various medical facilities and regions around the country. In the present study, the PCI method and patient outcomes were reviewed and compared following PCI to LMT using either a bare metal stent (BMS) or a DES at 4 hospitals performing PCI in Fukushima City.

\section{METHODS}

\section{Subjects}

The study population consisted of 155 consecutive patients (age : $69.7 \pm 10.6$ years) who underwent LMT lesion stent placement for angina pectoris (AP) or AMI between January 2008 and January 2013 at 4 hospitals in Fukushima City (Fukushima Medical University Hospital, Fukushima Red Cross Hospital, Saiseikai General Hospital, and Ohara Medical Center). Other than ACS subjects, each patient was received explanation in detail from the physicians for both CABG and PCI in prior to each treatment. After being given thorough information including risks, advantages, and disadvantages of both procedures, only patients who chose PCI treatment rather than $\mathrm{CABG}$ were enrolled in this study. Out of the 155 consecutive patients, 60 had ACS, and the remaining 95 had stable angina pectoris (SAP). Patients who were resuscitated due to shock before stent placement were excluded from the study. Coronary angiography findings were used to calculate each patient's Syntax score, which is an indicator of the complexity of coronary lesions as a whole. The Syntax score was developed as a combination of several previously validated angiographic classifications aiming to grade the coronary anatomy with respect to the number of lesions and their functional impact, location, and complexity ${ }^{12)}$. This score was initially used in SYNTAX (SYNergy between percutaneous coronary intervention with
TAXus and cardiac surgery) trial $^{10)}$, and then it has been widely used as a numerical value indicating the complexity of coronary lesions. High Syntax score represents the complex coronary lesions, lower score vice versa. BMSs were placed in 45 patients, and DESs were placed in 110 patients. Various factors were then compared between the BMS and DES groups. Written informed consent for each PCI was obtained from all patients. Data collection and clinical follow-up of the patients were approved by the ethical committee of Fukushima Medical University (No. 823 and No. 2002).

\section{Endpoint definitions}

The study endpoints were all-cause death and major adverse cardiac events (MACE) defined as cardiac death, AMI (CPK $\geq 3$ times reference value), hospitalization due to heart failure, and target lesion revascularization (TLR).

\section{Follow-up}

Chronic stage assessment was performed by gathering information on each clinical event from patient medical records at each hospital. For those who were not outpatients at any of the 4 hospitals, information was gathered by telephoning either the patient directly or the hospital to which the patient was transferred. All patients who developed clinical events were followed. Follow-up coronary angiography was performed on 107 patients at 6 to 10 months after stent placement. The post-coronary angiography follow-up rate was 69.0\% (107/155). Quantitative coronary angiography (QCA) and measurement of restenosis and stent expansion diameter were then performed to evaluate the site of the treated coronary artery. A semi-automated edgecontour-detection computer analysis system (Medis QCA CMS, version 7) was used for the QCA.

\section{Statistical analysis}

Continuous variables are expressed as mean \pm standard deviation (SD), and intergroup comparisons were done using Student's t-test. Survival curves were plotted using the Kaplan-Meier method, and event-free rates in each group were compared using the log-rank test. A P-value of $<0.05$ was considered significant. SPSS version 21.0 software (IBM, Armonk, NY, USA) was used to perform these statistical analyses. 


\section{RESULTS}

\section{Patient characteristics and LMT lesion morphology}

In consecutive cases in the registration period of this report, all patients were succeeded in PCI procedures, meaning that there was no intra- and peri-operative death and MACE. Table 1 shows the clinical characteristics of unprotected LMT patients who underwent stent treatment in the BMS and DES groups. Prior PCI was more common in the DES group, but no significant intergroup differences were observed for any of the other variables. Compared to their DES group counterparts, the BMS group patients had a higher rate of AMI $(33.3 \%$ vs. $10.9 \%)$ and a lower rate of SAP (35.6\% vs. $59.1 \%)$. The rate of emergent PCI was also significantly higher in the BMS group (53.3\% vs. 27.3\%). LMT lesion morphology was classified as follows : 1) ostium lesions, 2) mid-body lesions, 3) distal-body lesions, 4) bifurcation lesions of the left anterior descending (LAD) artery, and 5) bifurcation lesions of the left circumflex (LCx) artery. No significant intergroup differences were observed in any of these morphologies. Moreover, no differences were found in Syntax scores ${ }^{12)}$ (Table 2).

\section{Stent types and placement methods}

The stents used to treat patients in this study were BMS (29.1\%), DES (68.3\%), and a combination of both types (2.6\%). The following DESs were used : sirolimus-eluting stent (SES, Cypher ${ }^{\mathrm{TM}}$; $12.9 \%$ ), paclitaxel-eluting stent $\left(\mathrm{PES}\right.$, Taxus ${ }^{\mathrm{TM}}$; $11.6 \%$ ), zotarolimus-eluting stent (ZES, Endeavor $^{\mathrm{TM}} ; 1.9 \%$ ), everolimus-eluting stent (EES, Xience $^{\mathrm{TM}}$ or Promus ${ }^{\mathrm{TM}}$; $32.3 \%$ ), and biolimus-eluting stent (BES, Nobori ${ }^{\mathrm{TM}} ; 9.7 \%$ ) (Figure 1). BMSs and DESs, both of which were used in conjunction with a Y stent (culottes stenting), were simultaneously implanted in 2 patients. Single stents were placed in $91.1 \%$ of patients in the BMS group and $90.0 \%$ of patients in the DES group, and no significant differences were observed in the crossover stenting methods (Table 2). Approximately $10 \%$ of patients in each group were treated with 2 stents, primarily using the $\mathrm{Y}$ and $\mathrm{T}$ techniques, while just 1 patient in the DES group was treated with the crush technique. Intravascular ultrasound (IVUS) was used in more than $95 \%$ of cases in both groups. The kissing balloon technique (KBT) was also performed in approximately $75 \%$ of cases in both groups, with no significant intergroup difference. Distal protection (DP) and intra-aortic balloon pump (IABP) procedures were significantly more frequent

Table 1. Clinical characteristics of patients treated with BMS or DES.

\begin{tabular}{lccc}
\hline & BMS & DES & \\
& $n=45(\%)$ & $610(\%)$ & N.S. \\
\hline Age (years) & $69.4 \pm 11.2$ & $69.7 \pm 10.4$ & N.S. \\
Male & $38(84.4)$ & $89(80.9)$ & N.S. \\
Hypertension & $34(75.6)$ & $82(74.5)$ & N.S. \\
Diabetes Mellitus & $19(42.2)$ & $50(45.5)$ & N.S. \\
Dyslipidemia & $24(53.3)$ & $76(69.1)$ & N.S. \\
Smoking & $25(55.5)$ & $48(43.6)$ & N.S. \\
Obesity & $13(28.8)$ & $34(30.9)$ & 0.002 \\
Prior PCI & $11(24.4)$ & $55(50.0)$ & N.S. \\
Prior CABG & $0(0.0)$ & $1(0.9)$ & \\
\hline Diagnosis & & & 0.005 \\
AMI & $15(33.3)$ & $12(10.9)$ & N.S. \\
Unstable AP & $10(22.2)$ & $20(18.2)$ & N.S. \\
RMI & $1(2.2)$ & $2(1.8)$ & 0.007 \\
Stable AP & $16(35.6)$ & $65(59.1)$ & N.S. \\
Silent ischemia & $2(4.4)$ & $7(6.4)$ & N.S. \\
$\quad$ OMI & $1(2.2)$ & $4(3.6)$ & 0.004 \\
Emergent PCI & $24(53.3)$ & $30(27.3)$ & \\
\hline
\end{tabular}

PCI indicates percutaneous coronary intervention; CABG, coronary artery bypass grafting ; AMI, acute myocardial infarction ; AP, angina pectoris ; RMI, recent myocardial infarction; OMI, old myocardial infarction. 
Table 2. Lesion and Procedural Characteristics.

\begin{tabular}{|c|c|c|c|c|}
\hline & & $\begin{array}{c}\text { BMS } \\
n=45(\%)\end{array}$ & $\begin{array}{c}\text { DES } \\
n=110(\%)\end{array}$ & $P$ \\
\hline \multirow[t]{6}{*}{ Lesion } & Ostium & $6(13.3)$ & $11(10.0)$ & N.S. \\
\hline & Mid body & $9(20.0)$ & $16(14.5)$ & N.S. \\
\hline & Distal body & $11(24.4)$ & $33(30.0)$ & N.S. \\
\hline & Bifurcation LAD & $27(60.0)$ & 69 (62.7) & N.S. \\
\hline & Bifurcation LCx & $10(22.2)$ & $33(30.0)$ & N.S. \\
\hline & Syntax score & $19.0 \pm 8.1$ & $20.9 \pm 11.4$ & N.S. \\
\hline \multirow[t]{3}{*}{1 stent } & LMT single stent & $7(15.6)$ & $9(8.2)$ & N.S. \\
\hline & Cross over LCx & $29(64.4)$ & $72(65.5)$ & N.S. \\
\hline & Cross over LAD & $5(11.1)$ & $18(16.4)$ & N.S. \\
\hline \multirow[t]{3}{*}{2 stents } & Y stent & $2(4.4)$ & $7(6.4)$ & N.S. \\
\hline & $\mathrm{T}$ stent & $2(4.4)$ & $3(2.7)$ & N.S. \\
\hline & Crush stent & $0(0.0)$ & $1(0.9)$ & N.S. \\
\hline Stent size & Diameter, mm & $3.85 \pm 0.34$ & $3.46 \pm 0.17$ & $<0.001$ \\
\hline IVUS & $n(\%)$ & $44(97.8)$ & 105 (95.5) & N.S. \\
\hline $\mathrm{DP}$ & $n(\%)$ & $11(24.4)$ & $6(5.5)$ & 0.007 \\
\hline KBT & $n(\%)$ & 33 (73.3) & $84(76.4)$ & N.S. \\
\hline IABP & $n(\%)$ & 17 (37.7) & $16(14.5)$ & 0.005 \\
\hline PCPS & $n(\%)$ & $0(0.0)$ & $1(0.9)$ & N.S. \\
\hline
\end{tabular}

IVUS indicates intravascular ultrasound ; DP, distal protection ; KBT, kissing balloon technique ; IABP, intraaortic balloon pumping; PCPS, percutaneous cardio-pulmonary support.

\section{Types of stents}

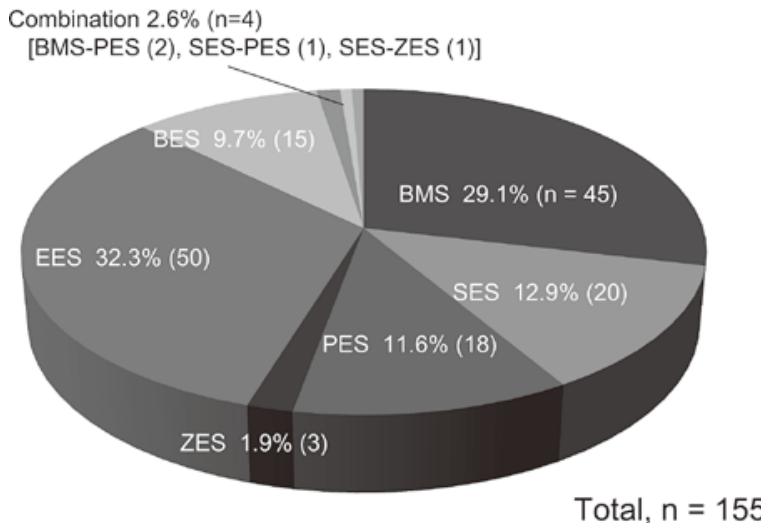

Fig. 1. Types of stents used in this survey. BMS bare metal stent ; SES, sirolimus-eluting stent ; PES, paclitaxel-eluting stent ; ZES, zotarolimuseluting stent; EES, everolimus-eluting stent ; BES, biolimus-eluting stent.

in the BMS group, but this was due to the high rate of BMS group patients with AMI at baseline. Mean stent diameter was significantly larger in the BMS group than in the DES group ( $38.5 \pm 0.34 \mathrm{~mm}$ vs. $3.46 \pm 0.17 \mathrm{~mm}, P<0.001)$. This was attributed to the fact that the sizes of DESs available for use in Japan are limited to diameters $\leq 3.5 \mathrm{~mm}$, compared to diameters of up to $4.0 \mathrm{~mm}$ for BMSs.

\section{Evaluation based on $Q C A$}

QCA was used to evaluate treated lesions in 107 patients (BMS group : 32 patients, DES group : 75 patients) who underwent confirmatory coronary angiography approximately 8 to 9 months (BMS group : $230.0 \pm 110.9$ days, DES group : $265.1 \pm$ 179.8 days) after initial treatment. Stent strut expansion diameter was significantly larger in the BMS group $(3.81 \pm 0.43 \mathrm{~mm}$ vs. $3.56 \pm 0.37 \mathrm{~mm}, P=$ 0.006 ), but this was simply due to the variation in stent sizes selected at treatment. The restenosis rate (\% stenosis), which is believed to be an indicator of neointimal proliferation, was significantly higher in the BMS group (26.6 $\pm 15.0 \mathrm{~mm}$ vs. 20.4 $\pm 12.6 \mathrm{~mm}, P=0.006$ ). This is a valid result when considering the respective pharmacological properties of BMSs and DESs.

\section{Long-term outcomes}

All patients who developed clinical events or no event were followed (follow-up rate : 100\%). The mean observation period for clinical events was $738.8 \pm 480.3$ days. MACE (all-cause death, ACS, heart failure, and TLR)-free rates for each group were compared (Figure 2A). As the figure illus- 

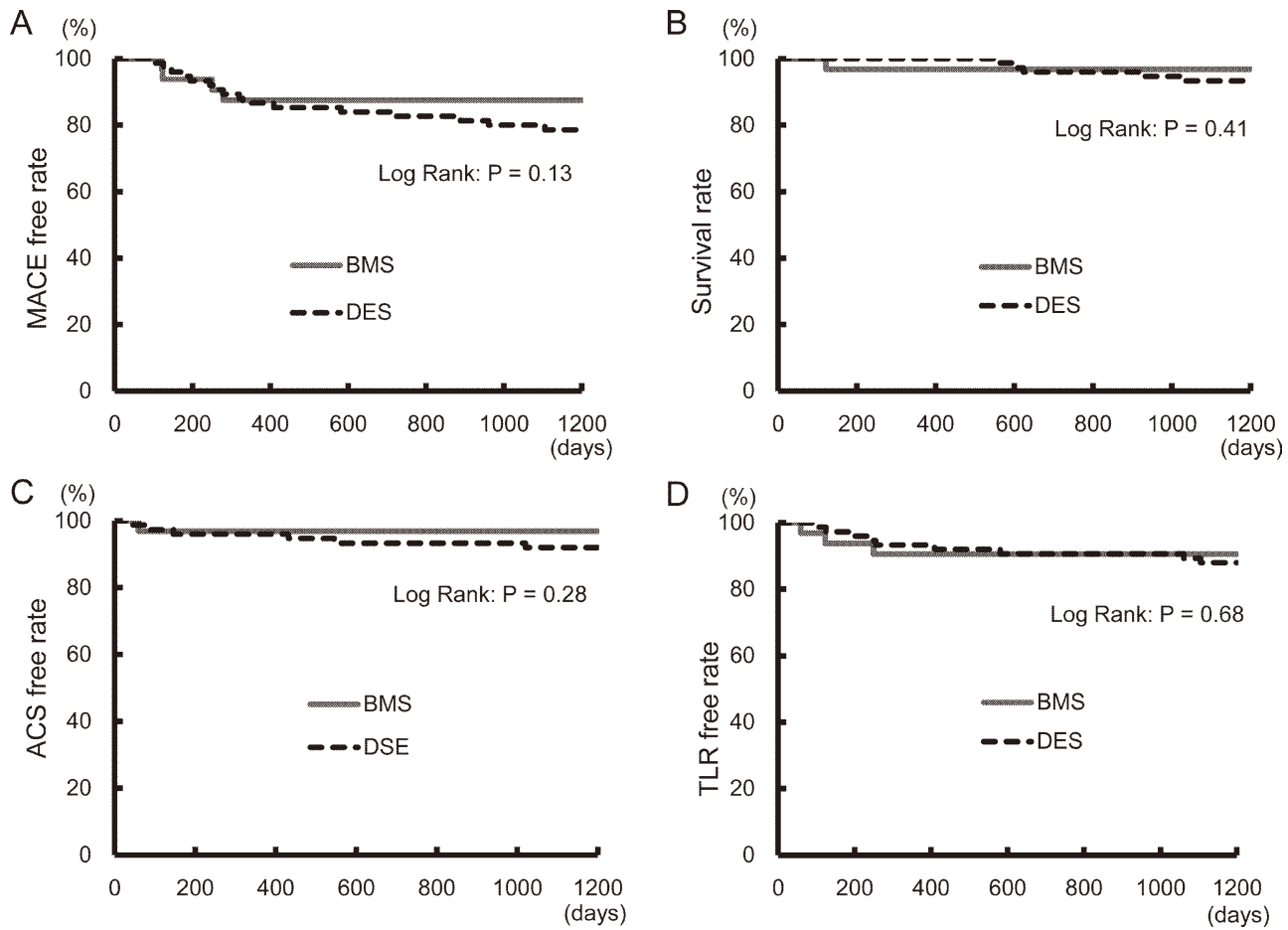

Fig. 2. Kaplan-Meier curves for event-free survivals. A Major adverse cardiac events (MACE)-free survival. B Cumulative all cause death-free survival. C Acute coronary syndrome (ACS)-free survival. D Target lesion revascularization (TLR)-free survival.

trates, no significant differences were evident between the 2 groups. All-cause death (Figure 2B), ACS (Figure 2C), and TLR (Figure 2D) were independently evaluated. All-cause death was seen in 1 case in the BMS group and in 5 cases in the DES group, of which 1 and 2 cases, respectively, were due to cardiac death. ACS was seen in 1 BMS group patient and 6 DES group patients, while TLR occurred in 3 BMS group patients and 9 DES group patients. No significant intergroup differences were observed in any of these study variables. The DES group was also analyzed according to first-generation stents (SES and PES) and second-generation stents (ZES, EES, and BES), but no significant differences were found.

\section{DISCUSSION}

Prior to 2012, the use of DESs to treat patients with AMI was not covered under health insurance in Fukushima Prefecture, so BMSs were often used in these patients. BMSs are associated with greater postoperative intimal proliferation than DESs, and it is a well-known fact that BMS use is accompanied by a high rate of TLR, which is caused by restenosis at the stented site $^{13)}$. On the other hand, a followup study conducted within 12 months of DES place- ment found that both restenosis and retreatment rates were very low, but late-onset ( $>12$ months) stent thrombosis was higher in DES than in BMS patients ${ }^{14)}$. Stent thrombosis is a crucial issue because it is responsible for a high rate of AMI. AMI due to LMT occlusion has an extremely high mortality rate compared to coronary occlusion in other areas ${ }^{15)}$. The use of stents with a high risk of lateonset stent thrombosis in the treatment of LMT lesions is therefore a controversial issue. However, the use of BMSs, with their higher restenosis and retreatment rates than DESs, is also controversial in the DES era. Nevertheless, DESs such as SES and PES have been shown to pose a higher risk of lateonset thrombosis and late-onset restenosis than BMSs from 12 months after placement ${ }^{16)}$, so if we could somehow limit the BMS drawback of restenosis and retreatment rates over the mid-term $(<12$ months), the use of BMSs to treat LMT lesions would become acceptable.

In the present study, there were no actual differences between the BMS and DES groups in allcause death or MACE onset after treatment (Figures 2). Endothelial proliferation in response to mechanical stress caused by stenting leads to narrowing of the vessel lumen, but this effect is more likely to occur in small vessels. Conversely, 
Table 3. Follow-up QCA Data of Both BMS and DES groups.

\begin{tabular}{llccc}
\hline & & $\begin{array}{c}\text { BMS } \\
\mathrm{n}=32\end{array}$ & $\begin{array}{c}\mathrm{DES} \\
\mathrm{n}=75\end{array}$ & $\mathrm{P}$ \\
\hline Follow up rate & $n(\%)$ & $32 / 45(71.1)$ & $75 / 110(68.2)$ & N.S. \\
Follow up period & days & $230.0 \pm 110.9$ & $265.1 \pm 179.8$ & N.S. \\
Stent strut diameter & $\mathrm{mm}$ & $3.81 \pm 0.43$ & $3.56 \pm 0.37$ & 0.006 \\
$\%$ stenosis & $\%$ & $26.6 \pm 15.0$ & $20.4 \pm 12.6$ & 0.040 \\
\hline
\end{tabular}

QCA indicates quantitative coronary angiography.

in vessels with a large diameter, restenosis is a rare occurrence, even when placing BMSs if the stent diameter is large. In LMT lesions, which were the target of the present study, the vessel diameter is usually the largest in the coronary artery, making it relatively advantageous for BMSs. In fact, the results of QCA measurement showed that the final expansion diameter after placement was significantly larger for BMSs than for DESs (Table 3).

Japanese treatment guidelines currently recommend the use of dual anti-platelet therapy (DAPT) after coronary stent placement for at least 1 month after BMS placement and at least 12 months after DES placement ${ }^{17)}$. Moreover, very late-onset stent thrombosis has been reported to occur after placement of a SES ${ }^{18)}$, which is a first-generation DES, so the prolonged continuous use of DAPT is recommended wherever possible in cases of placement in the LMT, in which there is a high risk when occluded. However, continued use of DAPT increases the risk of bleeding complications, and this bleeding risk is especially heightened when DAPT is combined with anticoagulant therapy for atrial fibrillation $^{19)}$. From this perspective, BMS treatment with short-term DAPT may be more suitable, particularly in patients with a high bleeding risk.

EES, ZES, and BES, so-called second-generation DESs used in recent years have a low incidence of late-onset and other stent thromboses ${ }^{20-22)}$. The present study included treatments performed between January 2008 and January $2013 ; 40.9 \%$ of the DESs used were first-generation, while the remaining $59.1 \%$ were second-generation. However, no significant differences in terms of either treatment outcome or prognosis were observed between the 2 generations of stents. Since global evidence has not yet to be obtained on the comparative incidences of late-onset stent thrombosis between second-generation DESs and BMSs, future studies are needed to evaluate the long-term efficacy and safety of second-generation DESs.

\section{STUDY LIMITATIONS}

Since this was not a prospective, randomized controlled trial, it is not possible to draw direct conclusions on the superiority of DES over BMS placement or vice versa. In addition, the study was limited to the local population of Fukushima City, so that the enrolled patient population was relatively small.

\section{CONCLUSIONS}

Late lumen loss associated with chronic intimal proliferation was frequent in the BMS group, but no significant difference was observed in terms of prognosis compared to the DES group. Considering the drawback of increased bleeding complications triggered by prolonged DAPT, we believe that use of a BMS is a viable option in the treatment of LMT lesions when it is possible to use a large-sized stent of more than $3.5 \mathrm{~mm}$ in diameter.

\section{CONFLICT OF INTEREST}

None of the authors has any conflicts of interest to disclose in relation to this investigation.

\section{REFERENCES}

1. A report from the ACC/AHA Task Force. Guidelines for percutaneous transluminal angioplasty. Circulation, 78 : 486-502, 1988.

2. O'keefe JH Jr, Hartzler GO, Rutherford BD, et al. Left main coronary angioplasty : early and late results of 127 acute and elective procedures. Am J Cardiol, 64 : 144-147, 1989.

3. Ellis SG, Tamai H, Nobuyoshi M, et al. Contemporary percutaneous treatment of unprotected left main coronary stenoses : initial results from a multicenter registry analysis 1994-1996. Circulation, 96 : 3867-3872, 1997.

4. Tan WA, Tamai H, Park SY, Plokker T, Nobuyoshi $\mathrm{M}$, Suzuki T, et al. Long-term clinical outcomes 
after unprotected left main trunk percutaneous revascularization in 279 patients. Circulation, 104 : 1609-1614, 2001.

5. ACC/AHA 2007 Guidelines for the management of patients with unstable angina/non-ST-elevation myocardial infarction : executive summary. Circulation, 116 : 803-877, 2007.

6. Guidelines for management of acute coronary syndrome without persistent ST segment elevation (JCS 2012) : web site publishing only (http://www. j-circ.or.jp/guideline/pdf/JCS2012_kimura_h.pdf)

7. Chieffo A, Stankovic G, Bonizzoni E, et al. Early and mid-term results of drug-eluting stent implantation in unprotected left main. Circulation, 111 : 791-795, 2005.

8. Valgimigli M, van Mieghem CA, Ong AT, et al. Short- and long-term clinical outcome after drugeluting stent implantation for the percutaneous treatment of left main coronary artery disease : insights from the Rapamycin-Eluting and Taxus Stent Evaluated At Rotterdam Cardiology Hospital registries (RESEARCH and T-SEARCH). Circulation, 111 : 1383-1389, 2005.

9. Park SJ, Kim YH, Lee BK, et al. Sirolimus-eluting stent implantation for unprotected left main coronary artery stenosis : comparison with bare metal stent implantation. J Am Coll Cardiol, 45 : 351-356, 2005.

10. Serruys PW, Morice MC, Kappetein AP, et al., for the SYNTAX investigators. Percutaneous coronary intervention versus coronary-artery bypass grafting for severe coronary artery disease. N Engl J Med, 360 : 961-972, 2009.

11. Guidelines for elective percutaneous coronary intervention in patients with stable coronary artery disease (JCS 2011) Published in 2012. Circ J, 77 : 1590-1607, 2013.

12. Sianos G, Morel MA, Kappetein AP, et al. The SYNTAX Score : an angiographic tool grading the complexity of coronary artery disease. EuroInterv, 1 : 219-227, 2005.

13. Stettler C, Wandel S, Allemann S, et al. Outcomes associated with drug-eluting and bare-metal stents : a collaborative network meta-analysis.
Lancet, 370 : 937-948, 2007.

14. Roukoz H, Bavry AA, Sarkees ML, et al. Comprehensive meta-analysis on drug-eluting stents versus bare-metal stents during extended followup. Am J Med, 122 : 581. e1-e10., 2009.

15. Nakazato K, Yamaki T, Kijima M, et al. Start and initial results of the Fukushima prefecture acute myocardial infarction registration survey. Fukushima J Med Sci, 59 : 27-34, 2013.

16. Daemen J, Wenaweser P, Tsuchida K, et al. Early and late coronary stent thrombosis of sirolimuseluting and paclitaxel-eluting stents in routine clinical practice : data from a large two-institutional cohort study. Lancet, 369 : 667-678, 2007.

17. Guidelines for management of anticoagulant and antiplatelet therapy in cardiovascular disease (JCS 2009) : web site publishing only (http://www.j-circ. or.jp/guideline/pdf/JCS2009_hori_h.pdf)

18. Nakazato K, Misaka T, Sakamoto N, et al. Worsening late-acquired incomplete stent apposition after sirolimus-eluting stent implantation for a chronic total occlusion lesion. Cardiovasc Intervent Ther, 30 : 85$91,2015$.

19. Dewilde WJM, Oirbans T, Verheugt FWA, et al., for the WOEST study investigators. Use of clopidogrel with or without aspirin in patients taking oral anticoagulant therapy and undergoing percutaneous coronary intervention : an open-label, randomised, controlled trial. Lancet, 381 : 11071115, 2013.

20. Palmerini T, Biondi-Zoccai G, Della Riva D, et al. Stent thrombosis with drug-eluting and baremetal stents. Lancet, 379 : 1393-1402, 2012.

21. Silber S, Windecker S, Vranckx P, et al. Unrestricted randomized use of two new generation drug-eluting coronary stents : 2-year patient-related versus stent-related outcomes from the RESOLUTE ALL Comers trial. Lancet, 377 : 1241-1247, 2011.

22. Natsuaki M, Kozuma K, Morimoto T, et al. Biodegradable polymer biolimus-eluting stent versus durable polymer everolimus-eluting stent. J Am Coll Cardiol, 62 : 181-190, 2013. 